\title{
Pour quelle raison Huizinga et Jolles se sont-ils séparés en 1933 ? Deux scénarios
}

Why Did Jolles and Huizinga Part Ways with Each Other in 1933? Two Scenarios

\section{Walter Thys}

\section{OpenEdition}

\section{Journals}

Édition électronique

URL : http://journals.openedition.org/cei/3260

DOI : $10.4000 /$ cei.3260

ISSN : 2260-779X

Éditeur

UGA Éditions/Université Grenoble Alpes

Édition imprimée

Date de publication : 30 décembre 2016

Pagination : 225-236

ISBN : 978-2-84310-337-7

ISSN : 1770-9571

\section{Référence électronique}

Walter Thys, "Pour quelle raison Huizinga et Jolles se sont-ils séparés en 1933 ? Deux scénarios », Cahiers d'études italiennes [En ligne], 23 | 2016, mis en ligne le 23 janvier 2017, consulté le 26 mars 2021. URL : http://journals.openedition.org/cei/3260 ; DOI : https://doi.org/10.4000/cei.3260 


\title{
POUR QUELLE RAISON HUIZINGA ET JOLLES SE SONT-ILS SÉPARÉS EN 1933 ? DEUX SCÉNARIOS ${ }^{1}$
}

\author{
Walter Thys $\dagger$ \\ Professeur émérite à l'Université Charles de Gaulle - Lille III \\ et à l'Université de Gand
}

\section{Introduction}

Dans sa courte autobiographie Mijn weg tot de historie, Huizinga décrit comme suit la fin brusque de son amitié avec André Jolles : "Meer dan vijf en dertig jaar heeft die vriendschap een groote plaats in mijn leven ingenomen, totdat zij op 9 October 1933 met één woord werd afgebroken, niet door $\mathrm{mij}^{2}$.»

Huizinga a en plus indiqué l'endroit où la séparation s'est produite, à savoir à la Gare Centrale d'Amsterdam. Et par son fils Leonhard Huizinga, dans Herinneringen aan mijn vader ${ }^{3}$, nous connaissons le contenu précis de leur dernière entrevue : «Huizinga: "Dan, André, valt er tussen ons eigenlijk niets meer te zeggen" waarop Jolles zei: "Nee, inderdaad niets

I. Le présent texte est la version légèrement raccourcie d'un article publié en néerlandais sous le titre "Waarom gingen Johan Huizinga en André Jolles in 1933 uit elkaar? ", Internationale Neerlandistiek, 52, I, 20I4, p. 67-8o.

2. [Mon chemin vers l'histoire], Haarlem, Tjeenk Willink, I947, p. 36 : «Pendant plus de trente-cinq ans cette amitié a pris une place importante dans ma vie, jusqu’à ce qu'elle fut brisée le 9 octobre I933 par un seul mot, ne venant pas de moi.» Pour des raisons de lisibilité, j’ai traduit en français des citations ou des termes en langues étrangères, aussi bien dans les notes que dans le texte.

3. [Souvenirs de mon père], La Haye, H. P. Leopold, 1963, p. I64: "Alors, André, entre nous il n'y a plus rien à se dire», ce à quoi Jolles aurait répondu : «Non, en effet, plus rien. »; «Et il monta dans le train et ne tourna même plus la tête.» Ce constat, par Huizinga lui-même, réfute l'hypothèse, répandue surtout en Allemagne, que c'était Huizinga qui avait rompu les relations parce qu'il ne voulait plus avoir de lien d'amitié avec un nazi. Même Bernd Roeck, dans son étude magistrale Florenz Igoo. Die Suche nach Arkadien (Munich, C. H. Beck Verlag, 2003 [200I]), p. 266, traduit l'opinion erronée que la rupture venait de Huizinga. La question de Huizinga, commençant par "Alors, André», et la réponse de Jolles, "Non, en effet" semblent être plutôt bizarres si l'on part de l'hypothèse que ce fut Huizinga qui rompit avec Jolles et non l'inverse. 
meer." ») Leonhard conclut ainsi : «En hij stapte in de trein en keek niet eens meer $\mathrm{om}^{4}$.»

Depuis pas mal de temps je me pose la question de savoir quelle peut bien être la cause de la brusque séparation en 1933 de ces deux savants après plus de trois décennies d'amitié. Dans l'exposé ci-dessous je tente à donner une réponse à cette question. Puisqu'une argumentation directe s'avère impossible par manque de documentation, j'ai dû recourir à différents scenarios afin d'en extraire une probable vérité.

\section{L'affaire von Leers, Leyde, le mardi in avril 1933}

Comme cela se savait dans les cercles universitaires néerlandais, il s'était produit ce jour-là un incident à l'Université de Leyde : le recteur de l'époque, le professeur Johan Huizinga, avait interdit l'accès à l'université à Johannes von Leers (1902-1965), guide de la délégation allemande à une conférence de l'International Student Service (ISS) qui avait lieu du 7 au I2 avril 1933. Il s'avérait que von Leers était l'auteur d'une brochure, Forderung der Stunde: Juden raus!'s (1928 et 1933), contenant un passage concernant le soi-disant meurtre rituel accompli par des Juifs sur des enfants chrétiens. La presse néerlandaise a dû être au courant de la brochure récusée dès le début de la conférence ${ }^{6}$. De ce fait, Huizinga n'aurait pas dû attendre jusqu'au II avril, l'avant-dernier jour du congrès, pour appliquer les mesures adéquates. Ou n’a-t-il pas voulu faire échouer le congrès dès son début ${ }^{7}$ ?

\section{La vengeance de von Leers}

Tout porte à croire que von Leers, après ce qu'il a dû ressentir comme un affront public, soit rentré en Allemagne avec des sentiments de vengeance. L'incident a fait l'objet d'un remarquable rapport par le professeur Willem Otterspeer, dont rien que le titre Huizinga voor de afgrond [Huizinga devant

4. Leonhard Huizinga situe la rupture "dans la deuxième partie des années trente", mais il ajoute qu'il ne sait pas retenir des dates.

5. [Exigence de l'heure : les Juifs dehors!']

6. Voir le journal Het Vaderland du samedi 8 avril ı933, édition du soir : «Het Nationaal-Socialisme en de Joden" [Le national-socialisme et les Juifs], premier article de la première page.

7. Ni les rédacteurs de la correspondance de Huizinga (J. Huizinga, Briefwisseling, II, Utrecht, Anvers, Tjeenk Willink, 1990, p. 434, 436, n. 5) ni le professeur Otterspeer n'ont vraisemblablement pas eu connaissance du numéro en question de Het Vaderland. 
l'abîme] provoque un frisson... Mais lorsqu'on se fait «l'avocat du diable», on peut se faire une meilleure idée de l'état d'esprit de ce nazi : en tant que confident de Goebbels, Himmler et Rosenberg et en tant que biographe de Hitler et auteur de plusieurs livres de propagande, subir un tel affront de la part d'un recteur d'université devant une délégation importante — plus de quarante participants - d'une toute jeune Allemagne nazie lors d'un congrès international, est une humiliation dont il a sans le moindre doute voulu se venger. En effet, dès son retour en Allemagne, toute une série de mesures ont été prises contre Huizinga, parmi lesquelles l'interdiction de la publication en Allemagne de ses travaux en cours, un Einreiseverbot [défense d'entrer en Allemagne] et un Vortragsverbot [défense de faire des conférences], l'ajout de ses ouvrages à la liste des schädlichen und unerwünschten Schrifttums [liste des livres nuisibles et non-désirables], mesures auxquelles on pourrait ajouter celles prises à partir de l'occupation des Pays-Bas par l'Allemagne en 1940 : la clôture de l'Université de Leyde, l'annulation de la nomination de Huizinga en tant que président de la Chambre des lettres de l'Académie royale néerlandaise des sciences, le licenciement de Huizinga en tant que professeur d'Université, son bannissement de Leyde et sa prise en otage dans un lieu situé dans la périphérie du pays. Finalement, dix ans après l'affaire de Leyde et deux ans avant le décès de Huizinga, un auteur allemand, Hellmut Schramm, fait paraître un livre consacré à Der jüdische Ritualmord. Eine historische Untersuchung [Le meurtre rituel juif. Une recherche historique], une sorte de mise à jour du pamphlet de von Leers, dédié à Alfred Rosenberg et préfacé par von Leers. Dans son premier chapitre l'auteur écrit [traduction de l'allemand par W. T.] : "Encore récemment, en 1933, l'hospitalité de l'Université de Leyde a été refusée à Johann von Leers par le vieux Huizinga parce que le savant allemand [von Leers, W. T.] avait écrit également au sujet du meurtre rituel juif.» De cette façon — et cela est assez remarquable le nom de Huizinga, dans les dernières années de sa vie, a paru dans un livre qu'il aurait combattu avec la même fermeté que le pamphlet de von Leers sur le même sujet dix ans auparavant. Huizinga était encore en vie en 1943 mais on peut espérer qu'il n'ait pas eu connaissance de la parution de cette nouvelle étude. Cela vaut également pour une citation de von Leers, parue dans un livre de Steinbach, où il décrit Huizinga comme «der grösste Judenknecht und Hasser des Nationalsozialismus in Holland ${ }^{8}{ }$.

8. Voir M. Steinbach, "Friedrich Schneiders "Kaiserpolitik des Mittelalters" ", dans "Kämpferische Wissenschaft». Studien zur Universität Jena im Nationalsozialismus, U. Hossfeld et al. (Hg.), Cologne, Weimar, Vienne, Böhlau Verlag, 2003, p. 943-966, p. 950 ["le plus grand serviteur des Juifs et haïsseur du nationalsocialisme en Hollande. »] 
À cet arrière-plan il est intéressant et il semble même logique d'ajouter au compte de von Leers la rupture entre Huizinga et Jolles du 9 octobre 1933. Pour ce faire il manque, hélas, jusqu’à présent toute preuve certaine. Il reste certes remarquable que l'incident entre Huizinga et von Leers, ainsi que la rupture entre les deux amis, se sont situés dans la même année avec un intervalle de seulement quelques mois, et qu'en plus la suite des deux événements semble être également logique. Mais comme il n’y a pas de preuves, on ne peut, comme cela a déjà été souligné, se baser que sur des scénarios.

\section{Premier scénario}

\section{Leipzig, le dimanche 8 octobre 1933}

Comme il en avait l'habitude, André Jolles était assis à son bureau ${ }^{9}$ à l'Université de Leipzig, en train d'écrire sa lettre hebdomadaire à sa fille aînée Jeltje à Francfort-sur-le-Main. Jeltje Goldschmidt-Jolles venait de donner naissance à son troisième enfant, Ernst Walter, et elle avait demandé à son père quand il viendrait voir son dernier petit-fils. Jolles lui répondit :

Ob wir noch kommen? [...] Augenblicklich bin ich unabkömmlich. Es ändert sich bei der alten Universität wirklich Vieles. Der Geist der Studenten wendet sich immer mehr von der Wissenschaft ab — aber immer mehr einer Sache zu, die sie 'Schulung' nennen ${ }^{\mathrm{IO}}$.

Et un peu plus loin il ajoute :

[...] meine Söhne haben mich so bitter enttäuscht, dass ich das Bedürfnis habe, mit jungen Menschen, die zu mir kommen und vertrauen zu mir haben, Dinge durch zu führen, die für ihre Zukunft wichtig sind ${ }^{\text {II }}$.

Dans les heures ou les minutes qui ont suivi l'acte d'écrire et de poster sa lettre du dimanche, quelque chose de très important a dû se produire. Mais quoi? Jolles a pu recevoir la visite d'un ou deux de ses anciens étudiants auxquels il avait fait allusion dans sa lettre. Celui qui entre le premier

9. Là se trouvait également sa bibliothèque personnelle, et il y passait souvent la nuit.

Io. W. Thys, André Jolles (I874-1946) 'gebildeter Vagant'. Brieven en documenten, Amsterdam, Leipzig, Amsterdam University Press, Leipziger Universitätsverlag, 2000, p. 857. [ Si nous viendrons? Pour le moment je suis indispensable. Il y a vraiment beaucoup de choses qui changent dans la vieille Université. L'esprit des étudiants se détourne de plus en plus de la science pour se tourner vers quelque chose qu'ils appellent Schulung. » (scolarisation, instruction)]

II. Ibid. ["Mes fils m’ont tellement déçu que je sens la nécessité d'entreprendre, avec des jeunes gens qui viennent me voir et qui ont confiance en moi, des projets qui sont importants pour leur avenir.»] 
en ligne de compte est Wilhelm Spengler (I907-I96I) qui, à partir de I927, avait suivi les cours d'études germaniques, entre autres auprès de Jolles, avait été promu docteur avec une thèse consacrée à Schiller et, au moment des événements dont il est question, était professeur de gymnase à Leipzig. Il lui était dès lors extrêmement facile de rencontrer Jolles. Auparavant il avait rempli déjà bon nombre de fonctions et de missions auprès du parti et, peu de temps après la visite en question, il fut nommé collaborateur au Sicherheitsdienst. Plus tard, dans un rapport sur le professeur Jolles ${ }^{\mathrm{I} 2}$, il déclarera que depuis 193I il avait eu une relation très suivie avec lui. Dès lors, il est envisageable que Spengler ait appris confidentiellement au Parti Nazi que (à l'instigation de von Leers?) l'on allait prendre des mesures disciplinaires contre Jolles si ce dernier ne rompait pas stante pede avec son ami Huizinga ${ }^{13}$. Jolles se voyait donc obligé de partir immédiatement pour la Hollande afin d'avoir une dernière entrevue avec son ami de plus de trente ans et de lui apprendre qu'il mettait fin à leur amitié. Très probablement il prit cette décision sans mettre au courant les membres de sa famille - il avait l'habitude d'agir de cette façon. Et il renonça également à un détour par Francfort où il aurait pu rendre visite à son petit-fils nouveau-né. Spengler lui aura fait comprendre qu'il était dans son propre intérêt d'agir aussi discrètement que possible (ce qui explique que dans la correspondance ultérieure de Jolles avec sa fille Jeltje on ne trouve nulle part une allusion, ni au voyage, ni à la rencontre avec Huizinga) ${ }^{\mathrm{I}}$. Ce que Jolles d'autre part devait faire sans tarder, c'était de téléphoner ou de télégraphier à Huizinga pour être sûr que son ami soit chez lui et en mesure de l'accueillir.

Une des définitions du terme "scénario" est «le déroulement supposé ou prévu à l'avance d'un ou de plusieurs événements. » C'est la définition qui s'applique ici. Cependant, un scénario perd sa validité lorsqu' un autre, plus crédible, se manifeste. Eh bien, le hasard a voulu qu'en effet un autre déroulement des événements, inconnu et inattendu, s'est produit, dans

I2. Ibid., p. $874-875$.

13. Dans cette hypothèse on peut également supposer que Jolles avait reçu d'une haute autorité (von Leers?) l'ordre de détruire, ou bien de livrer pour destruction, les lettres qu'il avait reçues de Huizinga — il s'agissait d'environ deux cents missives; de là proviendraient les recherches vaines, depuis maintenant près de trois quarts de siècle, de la moitié la plus précieuse de leur correspondance.

I4. Exactement à partir de ce jour un nouvel horaire ferroviaire international était entré en vigueur, grâce auquel à $22 \mathrm{~h} 07$ un train partait de Leipzig pour arriver à Amsterdam le jour suivant à $9 \mathrm{~h} 05$. C'était tout de même une durée du trajet simple de onze heures (je tiens à remercier sincèrement M. Lansink des Archives de la ville d'Utrecht, qui a bien voulu contrôler les horaires dans le Guide officiel des chemins de fer néerlandais à partir du 8 octobre 1933). 
lequel, en dehors de Huizinga et de Jolles, un troisième acteur entre en scène : Jan Andries, un des deux fils d'André Jolles.

\section{Deuxième scénario}

\section{Le troisieme homme}

Parmi les six enfants issus du mariage d'André Jolles avec l'Allemande Mathilde Mönckeberg, le fils aîné Jan Andries (Fribourg-en-Brisgau 1906 - Quito [Équateur] 1942) était de loin le plus aventureux. Cela se manifesta déjà dans ses premières années : défier le danger, monter des chevaux à cru, se balader sur la gouttière de son école à la recherche de son crayon, franchir clandestinement des frontières, etc. De son métier il était relieur. À la recherche d'un travail, à partir de Leipzig où il vivait avec son père et la deuxième femme de ce dernier, il arriva à Amsterdam, mais n'en trouvant pas, il décida de se diriger vers l'Angleterre, «la Terre promise des relieurs». Pour une raison inconnue, il renonça à son projet et embarqua précipitamment pour l'Uruguay. À partir de là il arriva clandestinement en Argentine où il se présenta sans détour au siège du Parti communiste, selon ses dires en tant que représentant de la jeunesse communiste des Pays-Bas. Pendant plusieurs années il a effectué clandestinement, et dans la plupart des cas bénévolement, plusieurs missions politiques pour le parti, au cours desquelles il a été arrêté à plusieurs reprises. Entre-temps il avait épousé une compañera indigène qui lui fit deux enfants. Le ménage vivait toutefois dans des circonstances difficiles. Arrêté une fois de plus en 1931, il fut condamné à dix-huit mois de prison, après quoi, le $\mathrm{I}^{\mathrm{er}}$ avril 1933 , l'Argentine le livra en tant qu'étranger illégal à l'Allemagne ${ }^{\mathrm{Is}}$.

Il était persuadé qu'en débarquant à Hambourg il serait immédiatement conduit dans un camp de concentration, mais cette perspective ne lui inspirait pas la moindre peur. Cependant, lors de son arrivée à Hambourg, le 29 avril 1933, il lui fut permis de quitter librement le navire et en peu de temps il rejoignait sa mère qui habitait la même ville. Personne, même pas lui, ne comprenait comment cela put se produire. Est-il pensable que son père (à sa fille Jeltje, le 28 mai 1933 : «Ich habe getan was ich glaubte tun zu müssen $\left.{ }^{16} »\right)$ ait pu obtenir du Parti que Jan ne soit pas arrêté, à condition de quitter immédiatement le pays? C’est concevable,

I5. Tout au long du voyage, il restait sous la stricte surveillance du capitaine, et à l'approche des ports il était enfermé dans sa cabine.

I6. ["J'ai fait ce que j'estimais devoir faire.»] 
et c'est peut-être également la raison pour laquelle Jan voulait se rendre en Espagne. Mais la cellule communiste de Hambourg avec laquelle il prit contact fit savoir à Moscou qu' elle se méfiait de lui. Comme il y avait aussi une menace provenant de la droite politique — selon la SA à Hambourg, son père, ainsi que les autres membres proches de sa famille, étaient disposés à livrer des informations à son sujet ${ }^{17}$ — il pria le banquier hambourgeois Ernst Sachs, un neveu de sa mère par alliance, de lui procurer un faux passeport. Celui-ci portait le nom de Manuel Enrique Casson Arribar, né en 1903 dans la localité de Tararon en Espagne de parents espagnols, Gilberto Casson et Julia Arribar ${ }^{18}$. À ce moment, Jan envoya des télégrammes chiffrés à Moscou, priant de lui envoyer un visa pour l'Union soviétique. Quelque temps après, Moscou a dû l'informer d'une menace imminente d'arrestation et lui ordonne de renoncer à son voyage en Espagne et de se rendre sans tarder en Union soviétique. Puisque Jan parlait parfaitement l'espagnol, et que même en s'exprimant en allemand il avait un accent espagnol très prononcé, il se procura sans problèmes, en tant que "touriste espagnol», un ticket pour Moscou auprès de l'Agence soviétique d'Intourist à Hambourg. Le Io août 1933, il entreprit le voyage sous sa nouvelle identité sans le moindre problème. Dans un premier temps, après son arrivée à Moscou, il eut quelques difficultés à cause de ses "déviations trotzkistes ${ }^{19}$ ", mais finalement il fut entendu par Sinani ${ }^{20}$, responsable pour l'Amérique latine auprès du quartier général de la Comintern, l'Internationale communiste (ou EKKI), fondée en I9I9 afin de répandre le communisme dans le monde, entre autres par la mission d'instructeurs (delegados en Amérique du Sud) rémunérés en dollars par Moscou. Jan Jolles, qui parlait aussi couramment le portugais, fut envoyé au Brésil où les communistes étaient en train de préparer un coup d'État. Dans la deuxième moitié de septembre 1933 Jan quitta l'Union soviétique — qu'il nommera dorénavant "sa patrie» — sous le nom de Manuel Casson (plus tard orthographié également Cazón) et vola en première classe pour le Brésil. Il fit escale à Leningrad, Helsinki, Copenhague, Harwich, Amsterdam, Bruxelles, Paris et ensuite Boulogne-sur-Mer, d'où son bateau partira pour l'Amérique du Sud.

I7. W. Thys, André Jolles (1874-1946) 'gebildeter Vagant', ouvr. cité, p. I07.

I8. Il n'y a aucune indication d'où provenaient les noms sur le passeport.

19. Voir Sinani dans une lettre du 8 septembre 1933 à un correspondant (ibid., p. I23-I24). Moscou, RGASPI 495/190/8I/70-7I. Étant soupçonné d'être un "provocateur trotskiste» et un "agent du service secret fasciste», Jan Andries Jolles fut exclu en 1938 du Parti communiste.

20. Pseudonyme de Georgi Wassiliewitch Skalov (I896-I936). Ensemble avec deux autres bonzes du parti, il préparait la révolution - manquée - de 1935 au Brésil. Il tomba en disgrâce et, lors de la première grande purge de 1936, il fut exécuté sommairement. 
Le voyage à travers l'Europe a duré presque deux mois et nous en sommes assez bien informés par les seize lettres que Jan a envoyées des pays visités à sa mère et à sa sœur cadette Ruth à Hambourg ${ }^{2 \mathrm{I}}$. Par ce moyen nous savons qu'il a séjourné à Amsterdam du 4 au I6 octobre 1933 et qu'il descendit à l'hôtel van den Berg, 58 Damrak. Dans une lettre à Ruth du dimanche 8 octobre, il l'informe que dans l'après-midi il ferait une promenade en voiture avec la famille Sachs qui avait fui Hambourg et s'était établie à Zandvoort, et que le jour d'après, le lundi 9 octobre, il entreprendrait une première visite "ganz systematisch" du Rijksmuseum.

\section{De retour à Leipzig, le dimanche 8 octobre 1933}

Ce jour-là, après avoir écrit et posté la lettre à sa fille Jeltje, André Jolles a pu recevoir d'elle un coup de téléphone ou un télégramme - elle était en contact régulier avec ses deux parents divorcés - lui annonçant que Jan, en route pour la France d'où il allait embarquer pour le Brésil, était de passage à Amsterdam, où il logeait à l'hôtel van den Berg au Damrak. Cette nouvelle a dû aviver la relation ambivalente que Jolles entretenait avec son fils ("In dieser Hinsicht war Jan zugleich mein Sohn und meine Karikatur $\left.^{22} »\right)$. Poussé par le regret, il a pu vouloir voir son fils une dernière fois afin de lui confesser qu'il se sentait responsable de son choix pour le communisme, puisque lui-même avait été communiste et avait éduqué son fils dans cette idéologie. En même temps il a pu penser qu'il pouvait le persuader de renoncer à sa mission pour le compte de la Comintern.

La rencontre entre un nazi et un communiste n'étant pas sans risques pour les deux, elle devait se produire dans le plus grand secret. André Jolles, ayant besoin d'un alibi pour entreprendre le voyage à Amsterdam, fit appel à l'aide de Huizinga. Apparemment il lui a immédiatement téléphoné, ou bien lui envoyé un télégramme afin de fixer un rendez-vous. Huizinga lui aura répondu que le lundi 9 octobre il serait très occupé par deux réunions à l'Académie royale des sciences, auxquelles non seulement il devait être présent mais qu'il devait présider. Huizinga lui a peut-être proposé une autre date mais Jolles avait une raison particulière pour ne pas remettre le rendez-vous : il ne savait pas combien de temps Jan Andries allait rester à Amsterdam — manifestement Jan lui-même l'ignorait — mais après les Pays-Bas il se serait rendu en Belgique et en France, et André Jolles,

2I. Ibid., p. I29-158.

22. ["En ce sens Jan fut en même temps mon fils et ma caricature.»] André Jolles à sa fille Jeltje dans une lettre du 9 février 1930 (ibid., p. 72I). 
à la recherche de son fils, craignait d'être arrêté dans un de ces deux pays à cause de sa condamnation à Gand en 1920 à quinze ans de travaux forcés par la Justice belge pour avoir collaboré à la Vlaamsche Hoogeschool de Gand (du point de vue juridique, sa crainte était infondée pour cause de prescription). De surcroît, ni en Belgique, ni en France André Jolles ne disposait d'amis ou de collègues pouvant intervenir en sa faveur.

\section{Amsterdam, le lundi g octobre 1933}

Lorsque Jolles, après un long voyage en train, arriva à l'hôtel van den Berg à Amsterdam, Jan s'avéra être absent ${ }^{23}$. Il se trouvait en effet au Rijksmuseum et après cette visite il a pu se rendre ailleurs. En théorie il est possible que Jan ait évité de son propre chef toute rencontre avec son père, ayant reçu de Moscou l'interdiction de parler à qui que ce soit dans certaines villes sur son trajet vers son lieu d'embarquement. Cependant, il est plus vraisemblable que Jan n'ait pas su que son père était à sa recherche car, lorsque plus tard, à Paris, il se trouvait avec son frère Matthijs, les deux ont eu plusieurs conversations dans lesquelles ils ont parlé de leur père d'une façon très négative, ce que Jan rapporta dans des lettres à sa mère et à sa sœur Ruth à Hambourg, mais sans la moindre mention d'un rendezvous - manqué — avec son père.

Dans ce deuxième scénario, tout comme dans le premier, nous sommes à la recherche de la raison pour laquelle Huizinga et Jolles se sont séparés en 1933. Cette fois-ci la réponse vient de l'extérieur. J'ai relu par hasard une correspondance que j'avais entamée entre 1971 et 1974 avec Mea NijlandVerwey (I892-I978), la fille du poète et professeur à l'Université de Leyde Albert Verwey. Je lui avais demandé si elle en savait plus sur la séparation entre Huizinga et Jolles. Voici sa réponse du 25 octobre 1972 [traduite du néerlandais par W. T.] :

Cette dernière visite de Jolles à Huizinga ne cesse de m’intriguer. A[ndré] J[olles] ne peut tout de même pas avoir espéré de pouvoir convertir J[ohan] H[uizinga] au Nazisme! Il doit lui avoir fait une demande à laquelle il pouvait espérer recevoir une réponse favorable, concernant des faits antérieurs, et que Huizinga a refusé. Mais comme à ce sujet il n'y a aucune preuve écrite, cela doit rester une supposition.

23. De toute évidence, André Jolles ne savait pas sous quel nom son fils était enregistré à l'hôtel. Un registre de réception aurait pu rendre service mais il n'a pas pu être trouvé. En réalité, Jan y était inscrit sous son propre nom de Jan Jolles : dès son arrivée à Amsterdam il avait envoyé une lettre avec son nom et son adresse à sa sœur Ruth pour qu'elle puisse lui écrire. 
Quelle demande concrète Jolles a-t-il pu faire à Huizinga le 9 octobre 1933, qui ait pu conduire à une rupture entre les deux amis? Il peut l'avoir prié de se servir de son influence (auprès des autorités? auprès de la Cour? - avec laquelle Huizinga entretenait des relations étroites) pour que Jan Jolles, se trouvant en Hollande avec un faux passeport, ne soit pas arrêté ni interné, et qu'avant tout il ne soit pas livré à l'Allemagne, car dans ce cas il serait sûrement envoyé dans un camp de concentration, et Jolles savait indubitablement ce que cela signifiait pour un communiste. $\mathrm{Ou}$ bien, Jolles a prié Huizinga de bien vouloir héberger son fils en secret pendant son séjour aux Pays-Bas afin de diminuer le risque qu’il soit arrêté; Huizinga a dû rejeter avec une fermeté et une indignation parfaitement admissibles, ainsi qu'avec un non possumus compréhensible, les demandes de Jolles ${ }^{24}$. Il ne fait aucun doute que des paroles très dures ont été échangées, qui ont finalement conduit à la rupture avec Huizinga, après quoi Jolles est retourné sans résultat à Leipzig. Le désarroi de Huizinga est connu.

\section{Conclusion}

Quoi qu'il en soit, ce lundi 9 octobre 1933 Johan Huizinga de Leyde, Jolles de Leipzig et Jan Andries Jolles alias Manuel Casson Arribar de Moscou, se trouvaient simultanément à Amsterdam. Cela ne peut indubitablement être contesté ou être imputé au hasard. Cependant, ce constat mène inéluctablement au rejet total du premier scénario, car il ne tient pas compte de la présence d'un troisième homme.

24. Ceci explique la réaction de Richard Roland Holst dans sa réponse du I2 octobre I933 à Huizinga concernant la lettre de ce dernier au sujet de ce qui s'était passé : «Qu’est-ce qui lui a pris [à André Jolles, W. T.] de t'inciter d'abord à le rencontrer et ensuite, après la rencontre, de rompre?» ( J. Huizinga, Briefwisseling II, ouvr. cité, p. 486.) 


\section{Bibliographie}

CoHen Adolf Emile, "Huizinga als Leids hoogleraar", Bijdragen en Mededelingen betreffende de Geschiedenis der Nederlanden, 88, 1973, p. 335-347.

Hossfeld Uwe et al. (éd.), "Kämpferische Wissenschaft». Studien zur Universität Jena im Nationalsozialismus, Cologne, Weimar, Vienne, Böhlau Verlag, 2003.

Huizinga Johan, "Burgund, eine Krise des Romanisch-Germanischen Verhältnisses", Historische Zeitschrift, CXLVIII, I, I933, p. I-28.

Huizinga Johan, Mijn weg tot de historie, Haarlem, Tjeenk Willink, 1947. Huizinga Johan, Briefwisseling II, Utrecht, Anvers, Tjeenk Willink, I990. Huizinga Johan, Das Spätmittelalter, Turnhout, Brepols («Millennium. Tijdschrift voor middeleeuwse studies"), 25, I, 201 I.

Huizinga Leonhard, Herinneringen aan mijn vader, La Haye, H. P. Leopold, 1963.

Jolles André, Einfache Formen. Legende, Sage, Mythe, Rätsel, Spruch, Kasus, Memorabile, Märchen, Witz, Tübingen, Max Niemeyer, I930.

von Leers Johann, Forderung der Stunde: Juden raus! Berlin, P. Hochmuth, 1933 (1928).

Otterspeer Willem, Huizinga voor de afgrond. Het incident-Von Leers aan de Leidse universiteit in I933, Utrecht, HES, 1984.

Roeck Bernd, Florenz I9oo. Die Suche nach Arkadien, Munich, C. H. Beck Verlag, 2003 (200I).

Schramm Hellmut, Der jüdische Ritualmord. Eine historische Untersuchung, Berlin, Theodor Fritsch Verlag, 1943.

Steinbach Matthias, "Friedrich Schneiders "Kaiserpolitik des Mittelalters" ", dans "Kämpferische Wissenschaft». Studien zur Universität Jena im Nationalsozialismus, ouvr. cité, p. 943-966.

Thys Walter, André Jolles (I874-I946) 'gebildeter Vagant'. Brieven en documenten, Amsterdam, Leipzig, Amsterdam University Press, Leipziger Universitätsverlag, 2000.

Thys Walter, «Waarom Huizinga en Jolles in 1933 uit elkaar gingen. Een scenario ", dans Id., Intra \& Extra Muros. Verkenningen voornamelijk in de neerlandistiek en het comparatisme, Delft, Eburon, 2008, p. 370-397.

Thys Walter, Vom Wandervogel zum 'Compañero'. Jan Andries Jolles (I906-1942). Soldat der Weltrevolution, Leipzig, Leipziger Universitätsverlag, 2012.

Thys Walter, «Waarom gingen Johan Huizinga en André Jolles in 1933 uit elkaar?", Internationale Neerlandistiek, 52, 20I4, p. 67-80. 


\section{Biographie de Walter Thys}

Walter Thys (1924-2015) a été professeur émérite des universités Charles de Gaulle - Lille III et de Gand. Il a publié la première biographie d'André Jolles ("Uit het leven en werk van André Jolles», dans De Nieuwe Taalgids, 1954) et il est l'éditeur de la correspondance d'André Jolles (2000) et de Jan Jolles (20I2). Il a prononcé le discours inaugural à l'occasion du centenaire de la naissance de Huizinga à l'Université de Groningue en 1972. 